Pacific Journal of Mathematic 


\title{
ON THE HANDLEBODY DECOMPOSITION ASSOCIATED TO A LEFSCHETZ FIBRATION
}

\author{
A. KAS
}

The classical Lefschetz hyperplane theorem in algebraic geometry describes the homology of a projective algebraic manifold $M$ in terms of "simpler" data, namely the homology of a hyperplane section $X$ of $M$ and the vanishing cycles of a Lefschetz pencil containing $X$. This paper is a first step in proving a diffeomorphism version of the Lefschetz hyperplane theorem, namely a description of the diffeomorphism type of $M$ in terms of "simpler" data.

Let $\tilde{M}$ be the manifold obtained from $M$ by blowing up the axis of a Lefschetz pencil. There is a holomorphic mapping $f: \tilde{M} \rightarrow \mathbf{C P}^{1}$ which is a Lefschetz fibration, i.e., $f$ has only nondegenerate critical points (in the complex sense). Using the Morse function $z \rightarrow|f(z)|^{2}$ on $\tilde{M}-f^{-1}(\infty)$, one obtains a handlebody decomposition of $\tilde{M}-f^{-1}(\infty)$ which may be described as follows: Let $X=f^{-1}(a)$ be a regular fiber of $f$. Choose a system of smooth ares $\gamma_{1}, \cdots, \gamma_{\mu}$ starting at $a$ and ending at the critical values of $f$ such that the $r$ 's are pairwise disjoint except for their common initial point. The $\gamma^{\prime}$ 's are ordered such that the tangent vectors $\gamma_{1}^{\prime}(0), \cdots$, $\gamma_{\mu}^{\prime}(0)$ rotate in a counter clockwise manner. To each $\gamma_{j}$ one may associated a "vanishing cycle", i.e., an imbedding $\phi_{j}$ : $S^{n} \rightarrow X(\operatorname{dim} X=2 n)$ defined up to isotopy, together with a bundle isomorphism $\phi_{j}^{\prime}: \tau \rightarrow \nu$ where $\tau$ is the tangent bundle to $S^{n}$ and $\nu$ is the normal bundle of $S^{n}$ in $X$ corresponding to the imbedding $\phi_{j}$. $\phi_{j}^{\prime}$ together with the well known bundle isomorphism $\tau \oplus \varepsilon \simeq \varepsilon^{n+1}$ determines a trivialization of the normal bundle of $e^{2 \pi i j} / \mu \times \phi_{j}\left(S^{n}\right)$ in $S^{1} \times X$. This trivialization allows one to attach a $n$-handle to $D^{2} \times X$ with the core $e^{2 \pi i j} / \mu \times \phi_{j}\left(S^{n}\right)$. If this is done for each $j, j=1, \cdots, \mu$, the resulting manifold is diffeomorphic to $\tilde{M}$-(tubular neighborhood of $f^{-1}(\infty)$ ).

Using the bundle isomorphism $\phi_{j}^{\prime}$ and the tubular neighborhood theorem one may identify a closed tubular neighborhood $T$ of $\phi_{j}\left(S^{n}\right)$ in $X$ with the tangent unit disk bundle to $S^{n}$. One may then define a diffeomorphism, up to isotopy, $\delta_{j}: X \rightarrow X$ with support in $T . \delta_{j}$ is a generalization of the classical Dehn-Lickorish twist. $\delta_{j}$ is the geometric monodromy corresponding to the $j$ th critical value of $f$. It follows that the composition $\delta_{\mu} \circ \cdots \circ \delta_{1}$ is smoothly isotopic to the identity $1_{X}: X \rightarrow X$. A smooth isotopy is given by a smooth arc $\lambda$ in $\operatorname{Diff}(X)$ joining the identity to $\delta_{\mu} \circ \cdots \circ \delta_{1}$. The choice of $i$, up to homotopy, determines the way in which one closes off $\tilde{M}$-(tubular neighborhood of $\left.f^{1}(\infty)\right)$ to obtain $\tilde{M}$. 
Thus the diffeomorphism type of $\tilde{M}$ is determined by the invariants $\phi_{1}, \phi_{1}^{\prime}, \cdots, \phi_{\mu}, \phi_{\mu}^{\prime}$ and $\{\lambda\}$, the homotopy class of $\lambda$. Conversely, given a compact oriented $2 n$ dimensional manifold $X$, imbeddings $\phi_{j}: S^{n} \rightarrow X, j=1, \cdots, \mu$, and bundle isomorphisms $\phi_{j}^{\prime}: \tau \sim \nu_{j}$ such that $\delta_{\mu} \circ \cdots \circ \delta_{1}$ is smoothly isotopic to $1_{X}$, and a homotopy class $\{\lambda\}$ of $\operatorname{arcs}$ in $\operatorname{Diff}(X)$ with initial point $1_{X}$ and end point $\delta_{\mu} \circ \cdots \circ \delta_{1}$, one may construct a $2 n+2$ dimensional manifold $\tilde{M}$ and a Lefschetz fibration $f: \tilde{M} \rightarrow \mathrm{CP}^{1}$. It is shown that in the case $n=1$, apart from certain exceptions, $\tilde{M}$ is uniquely determined by $\phi_{1}, \cdots, \phi_{\mu}$, i.e., the bundle isomorphisms $\phi_{1}^{\prime}, \cdots, \phi_{\mu}^{\prime}$ and the smooth isotopy class $\{\lambda\}$ are superfluous.

o. Introduction. The classical Lefschetz hyperplane theorem in algebraic geometry describes the homology groups of an algebraic manifold $M$ in terms of those of a hyperplane section $X$ and in terms of the "vanishing cycles" of $X$. This paper was inspired by the Morse theoretic proof of the Lefschetz hyperplane theorem due to Andreotti and Frankel [1]. Their approach is to blow up the base locus of a generic Lefschetz pencil so as to obtain a manifold $\widetilde{M}$ and a "Lefschetz fibration" $f: \widetilde{M} \rightarrow \boldsymbol{P}^{1}(\boldsymbol{C})$. They then use the Morse function $|f|^{2}$ to describe $\tilde{M}$, at least up to homotopy type, and finally they show how to relate the homology groups of $M$ to those of $\widetilde{M}$. Now according to Smale's handlebody theory [11], it should be possible to use the Morse function $|f|^{2}$ to determine the full diffeomorphism class of $\tilde{M}$, not just its homotopy type. In order to do this we must describe the framings of the imbedded spheres (vanishing cycles) corresponding to the critical points of $|f|^{2}$. In general, the framing associated to a critical point of index $n+1$, has an ambiguity measured by $\pi_{n}(S O(n+1))$. In our situation, we can improve this to $\pi_{n}(S O(n))$. This completely determines the framing in certain cases, most notably if $M$ is a complex surface.

In this paper, we describe a set of invariants associated to a Lefschetz fibration $f: M \rightarrow \boldsymbol{P}^{1}$, which allows one, in principle, to give a handlebody decompositions of certain complex surfaces.

There is a certain amount of overlap between some of the ideas of this paper and certain papers of B. Moishezon and R. Mandelbaum (cf. e.g., [6], [7], [10]).

1. The framings associated with a Lefschetz fibration. Let $M$ be a smooth manifold of dimension $\geqq 2$ and let $f: M \rightarrow S^{2}$ be a smooth mapping. A point $p \in M$ will be called a critical point of $f$ if the differential $d f_{p}: T(M)_{p} \rightarrow T\left(S^{2}\right)_{f(p)}$ is not surjective. Now let $M$ be a closed compact oriented smooth manifold of even dimension, 
say $\operatorname{dim} M=2 n+2, n \geqq 0$, and assume that $f: M \rightarrow S^{2}$ is a surjective mapping with a finite number of critical points. We will identify $S^{2}$ with the extended complex plane $C \cup \infty$. If $z \in S^{2}$ is a regular value of $f$, then $f^{-1}(z)$ is called a regular fiber of $f$. It is clear that up to diffeomorphism, the regular fibers of $f$ are independent of the regular value $z$.

Definition 1.1. The smooth mapping $f: M \rightarrow S^{2}$ will be called a Lefschetz fibration if each critical point $p$ of $f$ admits a coordinate neighborhood with complex valued coordinates $\left(w_{1}, \cdots, w_{n+1}\right)$, consistent with the given orientation of $M$, and if $f(p)$ has a coordinate neighborhood with a complex coordinate $z$, consistent with the orientation of $S^{2}$, such that locally, $f$ has the form:

$$
f(w)=z_{0}+w_{1}^{2}+\cdots+w_{n+1}^{2} .
$$

DEFinition 1.2. Two Lefschetz fibrations

$$
f_{1}: M \longrightarrow S^{2} \text {, and } f_{2}: M \longrightarrow S^{2}
$$

are said to be equivalent, if $f_{2}=g \circ f_{1}$ where $g$ is an orientation preserving diffeomorphism of $S^{2}$.

Let $X$ denote any regular fiber of the Lefschetz fibration $f: M \rightarrow S^{2}$. Notice that up to diffeomorphism, $X$ only depends on the equivalence class of the Lefschetz fibration $f: M \rightarrow S^{2}$. Notice also, that $X$ has a unique orientation consistent with the orientations of $M$ and $S^{2}$.

We wish to describe a handlebody decomposition of $M$ associated to the Lefschetz fibration $f: M \rightarrow S^{2}$. We will assume that a handlebody decomposition of $X$ is already known.

We first recall some standard facts about handles and handlebodies. Let $N$ be a manifold with boundary and let $n=\operatorname{dim} N$. Let

$$
\Phi: S^{k-1} \times D^{n-k} \longrightarrow \partial N
$$

be a smooth imbedding. Form the union $N_{1}=N \cup_{\phi} D^{k} \times D^{n-k}$ where we identify each point of $S^{k-1} \times D^{n-k} \subset \partial\left(D^{k} \times D^{n-k}\right)$ with its image under $\Phi . \quad N_{1}$ is a manifold with boundary and corner points. Let $V$ denote the unique manifold (possibly with boundary) obtained from $N_{1}$ by "straightening the corners" of $N_{1}$ (cf. [2]).

Definition 1.3. $\quad V$ is called: the manifold obtained from $N$ by attaching a $k$-handle along $\Phi$.

It is easy to see that, up to diffeomorphism, $V$ depends only on the smooth isotopy class of $\Phi$. Let $\Phi_{0}$ denote the restriction of 
$\Phi$ to $S^{k-1} \times 0$. It follows easily from the tubular neighborhood theorem [9], that $\Phi$ is determined, up to smooth isotopy, by a bundle isomorphism:

$$
\Phi^{\prime}: \varepsilon^{n-k} \longrightarrow \nu
$$

where $\varepsilon^{n-k}$ is the trivial $n-k$ bundle on $S^{k-1}$, and $\nu$ is the normal bundle of $S^{k-1}$ in $\partial N$ under the imbedding $\Phi_{0}$. Thus the isotopy class of the imbedding $\Phi$ is determined by:

(i) A smooth isotopy class of imbeddings

$$
\Phi_{0}: S^{k-1} \longrightarrow \partial N \text {; }
$$

(ii) For each $\Phi_{0}$ in (i), a smooth isotopy class of bundle isomorphisms:

$$
\Phi^{\prime}: \varepsilon^{n-k} \longrightarrow \nu .
$$

Notice that (i) is a "knot invariant". As for (ii), the distinct bundle isomorphisms, up to smooth isotopy, are classified by the group $\pi_{k-1}(S 0(n-k))$. $\Phi^{\prime}$ is called a framing of $\Phi_{0}$.

Let $F: M \rightarrow \boldsymbol{R}$ be a Morse function, and let $c \in \boldsymbol{R}$ be a critical value such that $F^{-1}(c)$ contains a single critical point $p$, where $F$ has index $\lambda$ at $p$. For each real number $a \in \boldsymbol{R}$, let $M_{a}=$ $\{x \in M \mid F(x) \leqq a\}$. Then for $\varepsilon>0$ sufficiently small, $M_{c+\varepsilon}$ is diffeomorphic to the manifold obtained from $M_{c-s}$ by attaching a $\lambda$-handle along $\Phi: S^{\lambda-1} \times D^{n-\lambda} \rightarrow \partial M_{c-\varepsilon}$. To construct $\Phi$ explicitely, one may choose (by Morse's lemma) a system of coordinates $x_{1}, \cdots, x_{n}$ in $M$ centered at $p$, such that $F\left(x_{1}, \cdots, x_{n}\right)=c-x_{1}^{2}-\cdots-x_{i}^{2}+x_{k+1}^{2}+\cdots$ $+x_{n}^{2}$ (cf. e.g., [8]). Then if $\xi=\left(\xi_{1}, \cdots, \xi_{\lambda}\right) \in S^{\lambda-1}, \eta=\left(\eta_{\lambda+1}, \cdots, \eta_{n}\right) \in$ $D^{n-\lambda}, \Phi: S^{\lambda-1} \times D^{n-\lambda} \rightarrow F^{-1}(c-\varepsilon)$ is defined by setting

$$
\Phi(\xi, \eta)=\left(x_{1}, \cdots, x_{n}\right)
$$

where

$$
\begin{aligned}
& \left(x_{1}, \cdots, x_{\lambda}\right)=\sqrt{\varepsilon+|\eta|^{2}} \xi \\
& \left(x_{\lambda+1}, \cdots, x_{n}\right)=\eta .
\end{aligned}
$$

Similarly, if $F^{-1}(c)$ contains several nondegenerate critical points $p_{1}, \cdots, p_{\ell^{\prime}}$ of indices $\lambda_{1}, \cdots, \lambda_{\mu}$, then $M_{c+s}$ is diffeomorphic to the manifold obtained from $M_{c-s}$ by attaching $/$ handles, where the $j$ th handle is a $\lambda_{j}$-handle attached along $\Phi_{j}: S^{\lambda_{j-1}} \times D^{n-\lambda_{j}} \rightarrow F^{1}(c-\varepsilon)$, $j=1, \cdots, \mu$, and where the images of the $\Phi_{j}$ are disjoint.

DEFINITION 1.4. A handlebody decomposition of a manifold $M$ is given by:

(i) a sequence $M=M_{N} \supset \cdots \supset M_{0}$, where each $M_{j}$ is a sub- 
manifold of $M$;

(ii) diffeomorphisms $\Phi_{j}: S^{\lambda_{j}-1} \times D^{n-\lambda_{j}} \rightarrow \partial M_{k}$, and

$$
\begin{aligned}
& \Psi_{j+1}: M_{j+1} \longrightarrow M_{j} \cup_{\Phi_{j}} D^{\lambda_{j}} \times D^{n-\lambda_{j}}, \\
& \Psi_{0}: M_{0} \longrightarrow D^{n} .
\end{aligned}
$$

A Morse function $F: M \rightarrow \boldsymbol{R}$ together with a gradient-like vector field for $F$, determine a handlebody decomposition of $M$ (cf. [6]).

Now let $f: M \rightarrow S^{2}$ be a Lefschetz fibration with regular fiber $X$, where $\operatorname{dim} X=2 n, \operatorname{dim} M=2 n+2$. We will assume that 0 and $\infty$ are regular values of $f$. Define $F: M \rightarrow \boldsymbol{R} \cup \infty$ by $F(p)=|f(p)|^{2}$. Then it is easy to verify that outside of $f^{-1}(0) \cup f^{-1}(\infty), F$ has only nondegenerate critical points, each of index $n+1$ (cf. [1]).

Definition 1.5. The Lefschetz fibration $f: M \rightarrow S^{2}$ is said to be normalized if the critical values of $f$ are precisely the $\mu$ roots of unity.

Every Lefschetz fibration is equivalent to a normalized Lefschetz fibration. Explicitely, if $a_{1}, \cdots, a_{t} \in S^{2}$ are the critical values of $f$, and if $a \in S^{2}$ is a regular value of $f$, choose $\operatorname{arcs} \gamma_{j}$ from $a$ to $a_{j}, j=1, \cdots, \mu$, which do not intersect except at $a$. Construct an orientation preserving diffeomorphism between a regular neighborhood of $\bigcup_{j=1}^{\mu} \gamma_{j}$ and the disk of radius $1+\varepsilon$ such that $a_{j}$ is mapped to $e^{2 \pi i j / \mu}$. Let $g: S^{2} \rightarrow S^{2}$ be an extension of this diffeomorphism. Then $g \circ f: M \rightarrow S^{2}$ is a normalized Lefschetz fibration.

Now assume that $f: M \rightarrow S^{2}$ is a normalized Lefschetz fibration, and let $D_{t} \subset S^{2}$ denote the disk of radius $t$ centered at the origin. Then $f^{-1}\left(D_{t}\right) \cong X \times D^{2}$ for $t<1$, while for $t>1, f^{-1}\left(D_{t}\right)$ is diffeomorphic to the manifold obtained from $X \times D^{2}$ by attaching $\mu$ $(n+1)$-handles by means of imbedding $\Phi_{j}: S^{n} \times D^{n+1} \rightarrow \partial\left(X \times D^{2}\right)=$ $X \times S^{1}, j=1, \cdots, \mu$, where the $\Phi_{j}$ have disjoint images.

LEMma 1.6. The imbeddings $\Phi_{j}$ may be chosen so that $\Phi_{j}\left(S^{n} \times 0\right) \subset f^{-1}\left(z_{j}\right)$ for some $z_{j}, j=1, \cdots, \mu$.

The proof of Lemma 1.6 will be given together with the proof of Theorem 1.7.

Let $\phi_{j}: S^{n} \rightarrow X$ be the imbedding defined by $\Phi_{j}$ restricted to $S^{n} \times 0$, and the identification $f^{-1}\left(D_{t}\right) \cong X \times D^{2}$, for $t<1$. Let $\nu_{1}$ denote the normal bundle of $S^{n}$ in $X$ corresponding to the imbedding $\phi_{j}$, and let $\nu$ denote the normal bundle of $S^{n}$ in $F^{-1}(1-\varepsilon)$. Clearly we have $\nu \cong \nu_{1} \oplus \varepsilon$. Let $\tau$ denote the tangent bundle of $S^{n}$.

THEOREM 1.7. There exists a bundle isomorphism $\phi_{j}^{\prime}: \tau \rightarrow \nu_{1}$. The framing $\phi_{j}^{\prime}: \varepsilon^{n+1} \rightarrow \nu$ coincides with the isomorphism. 


$$
\varepsilon^{n+1} \stackrel{\sim}{\longrightarrow} \tau \oplus \varepsilon \stackrel{\phi_{j}^{\prime} \oplus 1}{\longrightarrow} \nu_{1} \otimes \varepsilon \stackrel{\sim}{\longrightarrow \nu}
$$

Moreover $\phi_{j}^{\prime}$ is determined, up to smooth isotopy, by the local behavior of the function $f$ at the critical point $p$.

The proof of Lemma 1.6 and Theorem 1.7 are based on the following easy lemma.

LEMMA 1.8. Let $f: \boldsymbol{R}^{n} \rightarrow \boldsymbol{R}$ be a smooth function, and let $a=$ $\left(a_{1}, \cdots, a_{n}\right)$ be a regular point of $f$. Let $f(x)=f(a)+L(x-a)+$ $R(x-a)$ where $L(x)$ is a linear function and $|R(x-a)| \leqq k|x-a|^{2}$ for $|x-a|$ sufficiently small. Then for $\varepsilon>0$ sufficiently small, there exists a smooth function $F: \boldsymbol{R}^{n} \times I \rightarrow \boldsymbol{R}$ satisfying:

(i) $F(x, 0)=f(x)$;

(ii) $F(x, t)=f(x)$ for $|x-a|>\varepsilon$;

(iii) $F(x, 1)=f(a)+L(x-a)$ for $|x-a|<\varepsilon / 2$;

(iv) $x \rightarrow F(x, t)$ has no critical points in $\{|x-a|<\varepsilon\}$.

Proof. We may assume that $x=0$, and $f(a)=0$. Let $\rho_{\varepsilon}(s)$ be a smooth function such that

$$
\rho_{\varepsilon}(s)=\left\{\begin{array}{l}
1, s<\varepsilon / 2 \\
0, s>\varepsilon
\end{array} .\right.
$$

Let $F(x, t)=(t L(x)+(1-t) f(x)) \rho_{\varepsilon}(|x|)+f(x)\left(1-\rho_{\varepsilon}(|x|)\right)$. It is obvious that $F$ satisfies conditions (i), (ii), and (iii). To check condition (iv), we restrict our choice of $\rho_{\varepsilon}(s)$. Let $\rho(s)$ be a smooth function such that $\rho(s)=\left\{\begin{array}{l}1, s<1 / 2 \\ 0, s>1\end{array}\right.$. Then set $\rho_{s}(s)=\rho(s / \varepsilon)$. Let $M$ be a constant such that $\left|\rho^{\prime}(s)\right| \leqq M$. Then $\left|\rho_{\mathrm{s}}^{\prime}(s)\right| \leqq M / \varepsilon$. Letting $\vec{\nabla} f=\left(\partial f / \partial x_{1}, \cdots, \partial f / \partial x_{n}\right)$, and defining $F_{t}(x)=F(x, t)$ we have:

$$
\vec{\nabla} F_{t}(x)=-t \rho_{\varepsilon}(|x|) \vec{\nabla} R(x)-t R(x) \rho_{\varepsilon}^{\prime}(|x|) \frac{\vec{x}}{|x|}+\vec{\nabla} f
$$

where $\vec{x}=\left(x_{1}, \cdots, x_{n}\right)$. The first term on the right is small for small values of $\varepsilon$ since $\vec{\nabla} R(x) \rightarrow 0$ as $x \rightarrow 0$. As for the second term, we have $|R(x)|<k \varepsilon^{2}$ and $\left|\rho_{\varepsilon}^{\prime}(x)\right| \leqq M / \varepsilon$ for $|x|<\varepsilon$. Finally, $\vec{\nabla} f(0) \neq 0$ since 0 is a regular point. Therefore for $\varepsilon$ sufficiently small $\vec{\nabla} F_{t}(x) \neq 0$ for $|x|<\varepsilon$.

Proof of Lemma 1.6 and Theorem 1.7. Assume, for simplicity, that $j=1$. Since the Morse function $F$ is the composition of the Lefschetz fibration $w \rightarrow f(w)$, and the function $z \rightarrow|z|^{2}$, we can find, by Lemma 1.8, an arc of Morse functions $F_{t}: M \rightarrow R$ such that

(i) $F_{t}$ coincides with $F$ on $\{w \in M \| f(w)-1 \mid>\varepsilon\}$ 
(ii) $F_{0}=F$

(iii) $F_{1}(w)=2 \operatorname{Re} f(w)-1$ on $\{w \in M|| f(w)-1 \mid \leqq \varepsilon / 2\}$.

Since $F_{1}$ can be connected to $F_{0}$ by an arc of Morse functions, the handlebody decomposition associated with $F_{1}$ differs from that associated with $F_{0}$ by a smooth isotopy. Now if we use local complex coordinates $\left(w_{1}, \cdots, w_{n+1}\right)$ in a neighborhood of the critical point $p_{1}$ such that: $f\left(w_{1}, \cdots, w_{n+1}\right)=1+w_{1}^{2}+\cdots+w_{n+1}^{2}$, then $F_{1}(w)=2 \operatorname{Re} f(w)-$ $1=1+2|u|^{2}-2|v|^{2}$ where $w=u+i v, u, v \in \boldsymbol{R}^{n+1}$. Then $\Phi_{1}\left(S^{n} \times 0\right)=$ $\left\{\left.(u, v)|u=0,1-2| v\right|^{2}=1-\varepsilon\right\} \subset f^{-1}(\sqrt{1-\varepsilon})$, which proves Lemma 1.6.

To prove Theorem 1.7, consider the function: $g: S^{n} \times \boldsymbol{R}^{n+1} \rightarrow \boldsymbol{R}$ defined by $g(\xi, \eta)=\xi \cdot \eta \cdot g^{-1}(0)$ is the total space of the tangent bundle to $S^{n}$; thus if $\nu_{1}$ is the normal bundle of $S^{n} \times 0$ in $g^{-1}(0)$, then $\nu_{1}$ is naturally isomorphic to $\tau=\tau_{S^{n}}$. Since $g$ is a fibration, the normal bundle to $g^{-1}(0)$ in $S^{n} \times \boldsymbol{R}^{n+1}$ is trivial. Restricting these bundles to $S^{n} \times 0$, and using the standard metric on $S^{n} \times \boldsymbol{R}^{n+1}$, we get the splitting of bundles on $S^{n}, \varepsilon^{n+1} \cong \tau \oplus \varepsilon$. An easy computation shows that this coincides with the splitting induced by the standard imbedding of $S^{n}$ in $\boldsymbol{R}^{n+1}$. Now consider the diagram:

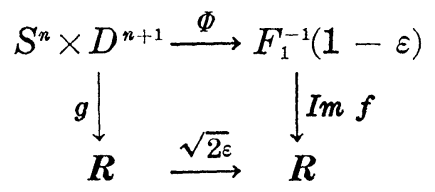

The diagram does not commute, but it does "commute to first order" on $S^{n} \times 0$. More precisely, this diagram induces a commutative diagram of bundle maps which proves Theorem 1.7.

DEFINITION 1.8. $\dot{\phi}_{j}\left(S^{n}\right) \subset X$ is called a vanishing cycle for the Lefschetz fibration $f: M \rightarrow S^{2}$. The bundle isomorphism $\phi_{j}^{\prime}: \tau \rightarrow \nu$ will be called a normalization of $\phi_{j}$. The pair $\tilde{\phi}_{j}=\left(\dot{\phi}_{j}, \phi_{j}^{\prime}\right)$ will be called a normalized vanishing cycle. The sequence of normalized vanishing cycles, $\left(\tilde{\phi}_{1}, \cdots, \tilde{\phi}_{n}\right)$ will be called an admissible sequence of normalized vanishing cycles.

Note that the bundle isomorphism $\phi^{\prime}: \tau \rightarrow \nu$ preserves or reverses orientations according to the factor $(-1)^{n(n-1) / 2}$. Thus, e.g., if $X$ is a real oriented surface, then a normalized vanishing cycle is completely determined by an imbedded circle. If $X$ is an oriented 4 manifold, the "core" of a normalized vanishing cycle is an imbedded $S^{2}$ with self intersection -2 .

Consider the case where $\operatorname{dim} M=4$, and therefore $X$ is an oriented surface. A vanishing cycle is then an imbedded circle in $X$, and the normalization of each vanishing cycle is unique. If 
$f: M, S^{2}$ is a Lefschetz fibration, then for a sufficiently large disk $G \subset S^{2}, f^{-1}(G)$ is diffeomorphic to the manifold obtain from $X \times D^{2}$ by attaching a finite number of 2-handles using attaching maps $\Phi_{j}: S^{1} \times D^{2} \rightarrow X \times S^{1}, j=1, \cdots, \mu$, where $\Phi_{j}\left(S^{1} \times 0\right)$ is an imbedded circle $\gamma_{j}$, in a fiber $X \times c_{j}$ of $X \times S^{1}$. Now there is a natural way to trivialize the normal bundle of $\gamma_{j}$ in $X \times S^{1}$ corresponding to the fact that the normal bundle of $\gamma_{j}$ in $X$ is trivial, and the normal bundle of $X$ in $X \times S^{1}$ is trivial. However, our Theorem 1.7 states that the framing $\Phi_{j}^{\prime}: \varepsilon^{2} \ldots \nu$ is obtained by identifying the normal bundle of $\gamma_{j}$ in $X$ with the tangent bundle $\tau$ of $\gamma_{j}$ and then using the isomorphism

$$
\varepsilon^{2} \cong \tau \oplus \varepsilon
$$

If one also pays attention to orientations, it is not hard to see that, relative to the "natural" framing, $\Phi_{j}^{\prime}: \varepsilon^{2} \rightarrow \nu$ has framing -1 . In other words, if we identify $\nu$ with $\varepsilon^{2}$ by the "natural" framing, then $\phi_{j}^{\prime}: \varepsilon^{2} \rightarrow \nu$ is given by the mapping $S^{1} \rightarrow S O(2) \cong S^{1}$ of degree -1 . This explains the well known fact (??) in algebraic geometry that relative vanishing cycles have self intersection number -1 .

2. Invariants of a Lefschetz fibration. Let $T S^{n}(1)$ denote the closed tangent unit disk bundle to $S^{n}$. We wish to describe a diffeomorphism

$$
\delta: T S^{n}(1) \longrightarrow T S^{n}(1)
$$

such that:

(i) $\delta$ is the identity on the boundary of $T S^{n}(1)$; and

(ii) $\delta$ is the antipodal map on $S^{n} \subset T S^{n}(1)$.

Let $(p, v) \in T S^{n}(1)$, where $p \in S^{n}$ and $v \in T S_{p}^{n}$ is a tangent vector of length $\leqq 1$. Form the geodesic arc on $S^{n}$ with initial point $p$, initial velocity $v$, and length $\pi|v|$, where $|v|$ denotes the length of $v$. Let $q$ be the end point, and $w$ the terminal velocity of this arc. Then we put $\delta(p, v)=\left(q^{*}, w^{*}\right)$ where $\left(q^{*}, w^{*}\right)$ is the image of $(q, w)$ under the antipodal map.

Now let $X$ be a closed manifold of dimension $2 n$; let $\phi: S^{n} \rightarrow X$ be an imbedding, and let $\phi^{\prime}: \tau \rightarrow \nu$ be a bundle isomorphism where $\tau$ is the tangent bundle to $S^{n}$, and $\nu$ is the normal bundle of $S^{n}$ in $X$. By the tubular neighborhood theorem, $\phi^{\prime}$ induces a diffeomorphism between $T S^{n}(1)$ and a tubular neighborhood of $\phi\left(S^{n}\right)$ in $X$. Thus we can apply $\delta$ to a tubular neighborhood of $\phi\left(S^{n}\right)$ in $X$, and after smoothing near the boundary, we can extend $\delta$ by the identity to a diffeomorphism of $X$ which we denote

$$
\delta_{\dot{\phi}, \phi^{\prime}}: X \longrightarrow X \text {. }
$$


Note that up to smooth isotopy $\delta_{\phi, \phi^{\prime}}$ only depends on the smooth isotopy class of the imbedding $\phi$ and the bundle isomorphism $\phi^{\prime}$. $\left(\phi, \phi^{\prime}\right)$.

Definition 2.1. $\delta_{\phi, \phi^{\prime}}$ is called the Dehn twist of $X$ with center

Notice that if $\operatorname{dim} X=2$, then $\delta_{\phi, \phi^{\prime}}$ is the classical right handed Dehn twist, i.e., $\delta_{\phi, \phi^{\prime}}$ maps the cylinder

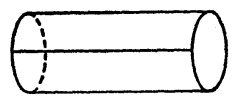

to

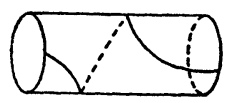

Now let $f: M \rightarrow S^{2}$ be a Lefschetz fibration with regular fiber $X$, and let $p \in M$ be a critical point of $f$. The part of $M$ which lies over the boundary of a small disk about $f(p)$ is then a fiber bundle over $S^{1}$ with typical fiber $X$. It is well known that such a fiber bundle is diffeomorphic to $X \times I /(x, 1) \sim(h(x), 0)$ where $h: X \rightarrow X$ is a diffeomorphism which is uniquely defined up to smooth isotopy. $h$ is the geometric monodromy of the fibering $f: M \rightarrow S^{2}$, associated with the critical value $f(p)$. Assume now that $p$ is the only critical point with critical value $f(p)$. Then as in $\S 1$, we may construct an imbedding $\phi: S^{n} \rightarrow X$, and normalization $\phi^{\prime}: \tau \rightarrow \nu$.

THEOREM 2.1 (cf. [3], page 148). The Dehn twist $\delta_{\phi, \phi}: X \rightarrow X$ is the geometric monodromy of $M$ about $f(p)$.

Proof. This theorem is proved in [3], but we will give another proof which is more consistent with our point of view. Let $I=$ $[-1,1]$ and fix an imbedding $\iota: I \rightarrow S^{1}$, e.g., $t \mapsto e^{i \varepsilon t}$. Now given a Lefschetz fibration $f: M \rightarrow S^{2}$ with critical value $f(p)$, choose a small circle $\gamma$ about $f(p)$; then $f^{-1}(\gamma)=Y$ is the manifold obtained from $X \times S^{1}$ by surgery. The surgery is performed as follows: look at $\phi^{\prime} \times \iota: T S^{n}(1) \times I \rightarrow X \times S^{1} . T S^{n}(1) \times I$ may be identified with $S^{n} \times D^{n}$ in a standard way (this involves straightening corners). The surgery consists of removing $S^{n} \times D^{n}=\phi^{\prime} \times \iota\left(T S^{n}(1) \times I\right)$ and sewing it back in by the automorphism of the boundary $S^{n} \times S^{n}$ given by $(\xi, \eta) \rightarrow(\eta, \xi) . \quad Y$ is given a differentiable structure by identifying $Y$ with $\left(X \times S^{1}-\phi\left(S^{n}\right) \times\{1\}\right) \cup D^{n+1} \times S^{n}$ where $\left(\xi^{\prime}, \eta^{\prime}\right) \in\left(D^{n+1}-0\right) \times S^{n}$ is identified with a point of $X \times S^{1}-\phi\left(S^{n}\right) \times\{1\}$ by the following map: first send $\left(\xi^{\prime}, \eta^{\prime}\right)$ to $(\xi, \eta)=\left(\xi^{\prime}|| \xi^{\prime}|,| \xi^{\prime} \mid \eta^{\prime}\right) \in S^{n} \times D^{n+1}$; then send $(\xi, \eta)$ to $(u, v) \times t \in T S^{n}(1) \times I$, and finally send this point to $\phi^{\prime}(u, v) \times$ 
$\iota(t)$. We may write

$$
Y=\left(X \times S^{1}-\dot{\phi}\left(S^{n}\right) \times\{1\}\right) \bigcup_{h} T S^{n}(1) \times I
$$

where $h: T S^{n}(1) \times I-S^{n} \times\{0\} \rightarrow X \times S^{1}-\phi\left(S^{n}\right) \times\{1\}$ is given as follows: if $(u, v) \times t \in T S^{n}(1) \times I-S^{n} \times\{0\}$, then $h((u, v), t)=\phi^{\prime}\left(u^{\prime}, v^{\prime}\right) \times \iota(t)$ where $\left(u^{\prime}, v^{\prime}\right)$ are given by:

$$
\begin{aligned}
& u^{\prime}=\frac{t}{\sqrt{t^{2}+|v|^{2}}} u+\frac{1}{\sqrt{t^{2}+|v|^{2}}} v \\
& v^{\prime}=\frac{|v|^{2}}{\sqrt{t^{2}+|v|^{2}}} u-\frac{t}{\sqrt{t^{2}+|v|^{2}}} v .
\end{aligned}
$$

Thus the vector $\left(\begin{array}{l}u^{\prime} \\ v^{\prime}\end{array}\right)$ is the product of the vector $\left(\begin{array}{l}u \\ v\end{array}\right)$ by the matrix:

$$
\begin{aligned}
\left(\begin{array}{cc}
\frac{t}{\sqrt{t^{2}+|v|^{2}}} & \frac{1}{\sqrt{t^{2}+|v|^{2}}} \\
\frac{|v|^{2}}{\sqrt{t^{2}+|v|^{2}}} & \frac{-t}{\sqrt{t^{2}+|v|^{2}}}
\end{array}\right)= & \left(\begin{array}{cc}
1 & 0 \\
0 & |v|
\end{array}\right)\left(\begin{array}{rr}
\cos \theta & \sin \theta \\
-\sin \theta & \cos \theta
\end{array}\right)\left(\begin{array}{ll}
0 & 1 \\
1 & 0
\end{array}\right) \\
& \times\left(\begin{array}{cc}
1 & 0 \\
0 & |v|^{-1}
\end{array}\right)
\end{aligned}
$$

where $\theta=\operatorname{arc} \sin t / \sqrt{t^{2}+|v|^{2}}$. These formulae show how the projection $X \times S^{1}-\phi\left(S^{n}\right) \times\{1\} \rightarrow S^{1}$ extends to a fibration $f: Y \rightarrow S^{1}$ where $f$ is defined on $T S^{n}(1) \times I$ by $f((u, v), t)=\iota(t)$.

The mapping $h: T S^{n}(1) \times I-S^{n} \times 0 \rightarrow T S^{n}(1) \times I-S^{n} \times 0$ given by $(u, v, t) \mapsto\left(u^{\prime}, v^{\prime}, t\right)$ has the following geometric description: Given $u \in S^{n}$ and $v \neq 0, v$ tangent to $S^{n}$ at $u$, define $\left(u^{\prime}, v^{\prime}\right)$ so that $u^{\prime}$ lies on the great circle through $u$ in the direction $v$ and the angle between $u$ and $u^{\prime}$ is $\theta+\pi / 2 . \quad v^{\prime}$ is tangent to the great circle, has the same length as $v$ and points backwards towards $u$. For fixed $t$, as $v$ approaches $0, \theta=\operatorname{arc} \sin t / \sqrt{t^{2}+|v|^{2}}$ approaches $\pi / 2$ for $t>0$ and $-\pi / 2$ for $t<0$. Thus $\left.h\right|_{S^{n} \times\{t\}}$ is the identity for $t<0$ and the antipodal map for $t>0$. For $|v|=1, \theta=\theta(t)$ goes from $-\pi / 4$ to $\pi / 4$ as $t$ goes from -1 to 1 .

Now to calculate the geometric monodromy of $f: Y \rightarrow S^{1}$, we construct a flow on $Y$. This is accomplished by constructing a smooth map $g: X \times[\varepsilon, 2 \pi+\varepsilon] \rightarrow Y$ such that $g(x, s) \in f^{-1}\left(e^{i z}\right)$ and $g_{s}: X \rightarrow f^{-1}\left(e^{i s}\right)$ is a diffeomorphism (the flow is $g_{*}(\partial / \partial s)$ ). The geometric monodromy is given by $g_{2 \pi+\varepsilon} \circ g_{\varepsilon}^{-1}$.

Identifying $Y$ with

$$
X \times S^{1}-\phi\left(S^{n}\right) \times\{1\} \bigcup_{h} T S^{n}(1) \times I
$$


we first define $g$ on $X \times[\varepsilon, 2 \pi+\varepsilon]-\phi^{\prime}\left(T S^{n}(1 / 2)\right) \times[2 \pi-\varepsilon / 2,2 \pi+\varepsilon]$ by setting $g(x, s)=\left(x, e^{i s}\right)$. We extend $g$ by a mapping $\widetilde{g}: T S^{n}(1) \times$ $I \rightarrow T S^{n}(1) \times I$ such that on $T S^{n}(1) \times I-T S^{n}(1 / 2) \times[-1 / 2,1] h \circ g=\mathrm{id}$. To construct $\widetilde{g}$, choose a smooth function $\zeta(|v|, t)$ such that: (i) $\zeta(|v|, t)=\theta(|v|, t)$ on $\{t \leqq-1 / 2\} \cup\{|v| \geqq 1 / 2\}$ and (ii) for fixed $t$, $\zeta(|v|, t) \rightarrow-\pi / 2$ as $|v| \rightarrow 0$. Now define $\widetilde{g}$ just as we defined $h$, but use $\zeta$ instead of $\theta$. Specifically,

$$
\widetilde{g}((u, v), t)=\left\{\begin{array}{l}
((u, v), t) \quad \text { if } \quad v=0 \\
\left(\left(u^{\prime}, v^{\prime}\right), t\right) \quad \text { if } \quad v \neq 0
\end{array}\right.
$$

where $u^{\prime}$ lies on the great circle through $u$ in the direction $v$, the angle between $u$ and $u^{\prime}$ is $\pi / 2+\zeta$, and $v^{\prime}$ points backwards towards $u$ along this geodesic and has the same length as $v$.

To calculate the monodromy $g_{2 \pi+\varepsilon} \circ g_{\varepsilon}^{-1}$, it is clear that the monodromy is concentrated on $T S^{n}(1) \subset X$, i.e., it is the identity outside of $T S^{n}(1)$. Notice that $g_{\varepsilon}$ is defined by the following diagram:

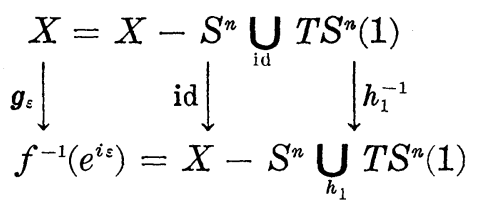

where $h_{t}=h_{T^{n}(1) \times t} \cdot g_{2 \pi+s}$ is defined by:

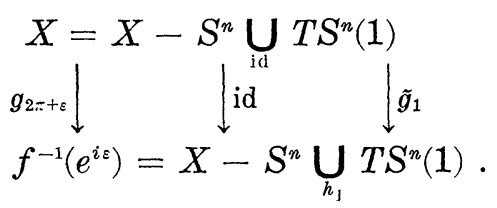

Thus the geometric monodromy $g_{2 \pi+\varepsilon} \circ g_{\varepsilon}^{-1}$ is concentrated on $T S^{n}(\mathbf{1}) \subset X$ and there it is equal to $\widetilde{g}_{1}^{-1} \circ h_{1}$. This mapping sends a point $(u, v) \in$ $T S^{n}(1)$ to $\left(u^{\prime}, v^{\prime}\right)$ where $u^{\prime}$ lies on the great circle through $u$ tangent to $v,\left|v^{\prime}\right|=|v|$, and the angle between $u$ and $u^{\prime}$ is $\dot{\phi}(|v|, 1)-\zeta(|v|, 1)$. This angle is a decreasing function of $|v|$, equal to $\pi$ for $|v|=0$ and equal to 0 for $|v|=1$. This map is therefore isotopic to the Dehn twist $\delta_{\phi, \phi^{\prime}}$.

Let $f: M \rightarrow S^{2}$ be a normalized Lefschetz fibration with fiber $X$, and critical values $e^{2 \pi i j^{\prime} \prime}, j=0,1, \cdots, \mu-1$. Let $E$ denote the subset of the disk of radius $1+\varepsilon$ obtained by removing the sets $E_{j}, j=1, \cdots, \mu$ where $E_{j}$ is described in polar coordinates $(r, \theta)$ by:

$$
E_{j}=\left\{(r, \theta) \mid 1-\varepsilon<r \leqq 1+\varepsilon, \frac{2 \pi j}{\mu}-\varepsilon<\theta<\frac{2 \pi j}{\mu}+\varepsilon\right\} .
$$


Identifying $f^{-1}(E)$ with $X \times E$, one may construct a vector field $\sigma$ on $f^{-1}\{|z|=1+\varepsilon\}$ such that $\sigma=(0, \partial / \partial \theta)$ outside of the $E_{j}$, while on $E_{j}$ the flow along $\sigma$ maps the fiber over $\theta=2 \pi j / \mu-\varepsilon$ to the fiber over $\theta=2 \pi j / \mu+\varepsilon$ by a Dehn twist $\delta_{j}, j=1, \cdots, \mu$. In particular, if we let $X_{\theta}$ denote the fiber over $\theta$, then the flow along $\sigma$ defines diffeomorphisms,

$$
\begin{aligned}
& h_{\theta}: X_{0} \longrightarrow X_{\theta} \text { such that, } \\
& h_{2 \pi}=\delta_{\mu^{\circ}} \cdots \circ \delta_{1} . \text { Since } f \text { has no critical }
\end{aligned}
$$

values outside the unit circle, the fibration defined by $f$ may be trivialized over the complement of $E$. In particular each fiber $X_{\theta}$ is then identified with $X=X_{0}$, and $\theta \rightarrow h_{\theta}$ may be regarded as an arc in $\operatorname{Diff}(X)$ joining the identity to $\delta_{\mu} \circ \ldots \circ \delta_{1}$. Any two such trivializations of the fibering $f$ are related by a diffeomorphism

$$
D^{2} \times X \longrightarrow D^{2} \times X
$$

of the form: $(z, x) \rightarrow\left(z, g_{z}(x)\right)$. The function $D^{2} \rightarrow \operatorname{Diff}(X)$ given by $z \rightarrow g_{z}$ gives a homotopy between the $\operatorname{arcs} \theta \rightarrow h_{\theta}$, corresponding to the two trivializations. Thus the homotopy class of the $\operatorname{arc} \theta \rightarrow h_{\theta}$ is an invariant of the normalized Lefschetz fibration $f: M \rightarrow S^{2}$.

THEOREM 2.3. The normalized Lefschetz fibration $f: M \rightarrow S^{2}$ is uniquely determined by: and

(1) a sequence of normalized vanishing cycles $\left(\tilde{\phi}_{1}, \cdots, \tilde{\phi}_{n}\right)$;

(2) a homotopy class of arcs $\theta \rightarrow h_{\theta}$ in $\operatorname{Diff}(X)$, where $h_{0}=\mathrm{id}_{X}$ and $h_{2 \pi}=\delta_{l^{\prime}} \circ \cdots \circ \delta_{1}$, where $\delta_{j}=\delta_{\bar{\psi}_{j}}$ is the Dehn twist of $X$ with center $\tilde{\phi}_{j}$.

Proof. This theorem is essentially a direct consequence of Theorem 1.7, and our above discussion. More precisely, the manifold $M$ and the fibration $f$ are constructed as follows: starting with $D^{2} \times X$, one attaches $\mu(n+1)$-handles along the centers $\Phi_{j 0}: S^{n} \rightarrow$ $\varepsilon_{j} \times X, \varepsilon_{j}=e^{2 \pi i j^{\prime} / \mu}$, where $\Phi_{j 0}$ coincides with $\phi_{j}: S^{n} \rightarrow X$, and the framing of $\Phi_{j 0}$ is given by the prescription of Theorem 1.7. The boundary of the resulting manifold fibers over a circle, with fiber $X$, and by Theorem 2.1, one may construct a vector field on this boundary whose flow gives rise to the diffeomorphism $\delta_{\mu} \circ \ldots \circ \delta_{1}$. The smooth arc $\theta \rightarrow h_{\theta}$ in $\operatorname{Diff}(X)$ joining $\mathrm{id}_{X}$ to $\delta_{\mu} \circ \cdots \circ \delta_{1}$ is then used to identify the boundary with $S^{1} \times X$, and we finally use this identification to close up the manifold by attaching a copy of $D^{2} \times X$.

The normalized vanishing cycle $\tilde{\phi}$ was defined (Def. 1.8) as a 
pair $\left(\phi, \phi^{\prime}\right)$ where $\phi: S^{n} \rightarrow X$ is an imbedding, and $\dot{\phi}^{\prime}: \tau_{S^{n}} \rightarrow \nu$ is a bundle isomorphism. $\phi$ and $\phi^{\prime}$ are defined up to smooth isotopy. The distinct bundle isomorphisms $\tau_{S^{n}} \rightarrow \nu$ are classified, up to isotopy, by the group $\pi_{n}(S O(n))$. Thus for those values of $n$ for which $\pi_{n}(S O(n))=0, \phi^{\prime}$ is uniquely determined. This is true, in particular, for $n=1$ and 2 .

Given an admissible sequence $\left(\tilde{\phi}_{1}, \cdots, \tilde{\phi}_{\mu}\right)$, let $M_{1}$ be the manifold with boundary obtained by attaching $\mu(n+1)$-handles to $D^{2} \times X$ as in Theorem 1.7. The distinct homotopy classes of arcs $\theta \rightarrow h_{\theta}$ in $\operatorname{Diff}(X)$ joining $\operatorname{id}_{X}$ to $\delta_{\mu} \circ \cdots \circ \delta_{1}$ are classified by $\pi_{1}(\operatorname{Diff}(X))$. Now a closed loop in $\operatorname{Diff}(X)$ gives rise to a fiber preserving diffeomorphism of $S^{1} \times X$, and a homotopy class corresponds to an isotopy class of such fiber preserving diffeomorphisms. Let $\left\{\theta \rightarrow h_{\theta}\right\},\left\{\theta \rightarrow h_{\theta}^{\prime}\right\}$ be two homotopy classes of $\operatorname{arcs} \operatorname{in} \operatorname{Diff}(X)$ joining $\operatorname{id}_{X}$ to $\delta_{\mu} \circ \ldots \circ \delta_{1}$ and $g: S^{1} \times X \rightarrow S^{1} \times X$ be a diffeomorphism representing their difference. If $g$ can be extended to a fiber preserving diffeomorphism of $M_{1}$, then the Lefschetz fibering, $f: M \rightarrow S^{2}$ associated to $h_{\theta}$ is equivalent to the Lefschetz fibration $f^{\prime}: M^{\prime} \rightarrow S^{2}$ associated to $h_{\prime \prime}^{\prime}$.

THEOREM 2.4. If $X$ is an oriented surface of genus $>1$, then every Lefschetz fibration $f: M \rightarrow S^{2}$ with fiber $X$ is uniquely determined, up to equivalence, by an admissible sequence of vanishing cycles. In case $X$ has genus 1 , the theorem is still true provided that $f: M \rightarrow S^{2}$ has at least one vanishing cycle $\phi: S^{1} \rightarrow X$ which is not homologous to zero.

Proof. It is clear that there is a unique normalization for any vanishing cycle in a surface (cf. the remarks on orientation, following Def. 1.8). The theorem in the case of genus $(X)>1$ follows from the fact that $\operatorname{Diff}(X)$ is contractible (cf. [4[). The case, genus $(X)=1$, follows from results of Moishezon [10] (Lemma 8 on page 179 and Lemma 7 on page 164).

It follows from Theorem 2.4, that to each equation of the form:

$$
\delta_{\mu \circ} \circ \cdots \circ \delta_{1}=1
$$

in the mapping class group of the oriented surface $X$, where $\hat{o}_{j}$ is the right-handed Dehn twist of $X$, centered at some circle $\gamma_{j}$ in $X$, one may associate a 4-manifold $M$ and a normalized Lefschetz fibration $f: M \rightarrow S^{2}$ whose critical values are the $\mu$ roots of unity and whose vanishing cycles are $\gamma_{1}, \gamma_{2}, \cdots, \gamma_{\mu}$. Let $g: S^{2} \rightarrow S^{2}$ be an orientation preserving diffeomorphism which leaves invariant the $\mu$ roots of unity. Then $f^{\prime}=g \circ f: M \rightarrow S^{2}$ is a normalized Lefschetz fibration with vanishing cycles $\gamma_{1}^{\prime}, \cdots, \gamma_{\mu}^{\prime}$. Following [10], page 180, we 
will say that the admissible sequence $\left(\gamma_{1}^{\prime}, \cdots, \gamma_{\mu}^{\prime}\right)$ is equivalent to $\left(\gamma_{1}, \cdots, \gamma_{\mu}\right)$. This relation is generated by the elementary transformations:

$$
T_{j}:\left(\gamma_{1}, \cdots, \gamma_{\mu}\right) \longrightarrow\left(\gamma_{1}^{\prime}, \cdots, \gamma_{k^{\prime}}^{\prime}\right)
$$

where:

$$
\begin{aligned}
\gamma_{j}^{\prime} & =\delta_{j}^{-1}\left(\gamma_{j+1}\right) \\
\gamma_{j+1}^{\prime} & =\gamma_{j} \\
\gamma_{k}^{\prime} & =\gamma_{k} \quad \text { if } \quad k \neq j, j+1
\end{aligned}
$$

(here the subscript $j$ is computed modulo $\mu$ ). The corresponding Dehn twists $\delta_{k}^{\prime}$ are given by:

$$
\begin{aligned}
\delta_{j}^{\prime} & =\delta_{j} \circ \delta_{j+1} \circ \delta_{j}^{-1} \\
\delta_{j+1}^{\prime} & =\delta_{j} \\
\delta_{k}^{\prime} & =\delta_{k} \text { if } k \neq j \text { or } j+1 .
\end{aligned}
$$

EXAMPLE 2.5. Let $X=T^{2}$ be a surface of genus 1, i.e., a 2-dimensional torus. Let $m$ denote a standard meridian circle, and let $l$ denote a standard longitude on $X$. Let $\left(\gamma_{1}, \cdots, \gamma_{\mu}\right)$ be an admissible sequence of imbedded circles on $X$, such that no $\gamma_{j}$ is homologous to zero. Then Moishezon has proved (cf. [10]) that $\mu \equiv 0(12)$ and $\left(\gamma_{1}, \cdots, \gamma_{\mu}\right)$ is equivalent to the sequence $(m, l, \cdots$, $m, l)$ ( $\mu$ terms).

3. Some open problems. In this section we will discuss several problems on Lefschetz fibrations of 4 -manifolds. Such fibrations arise naturally from Lefschetz pencils. Thus let $M$ be an algebraic surface imbedded algebraically in a complex projective space $\boldsymbol{P}^{N}$. Let $H^{N-2}$ be a generic linear space of codimension 2 in $\boldsymbol{P}^{N}$, and let $\widetilde{M}$ be the manifold obtained from $M$ by blowing up the points of $M \cap H^{N-2}$. Then there is a Lefschetz fibration $f: \widetilde{M} \rightarrow \boldsymbol{P}^{1}$ such that $f^{-1}(t) \cong M \cap L_{t}$, where $L_{t} \subset P^{N}$ is a hyperplane containing $H^{N-2}$ (such hyperplanes are naturally parametrized by $\boldsymbol{P}^{1}$ ). For the details of this construction ef [1]. By Theorem 2.4, the differentiable structure of $\widetilde{M}$ together with the Lefschetz fibration is completely determined by an admissible sequence of vanishing cycles. $M$ is obtained from $\widetilde{M}$ by blowing down certain exceptional curves. These exceptional curves are holomorphic sections of the fibration $f: \tilde{M} \rightarrow \boldsymbol{P}^{1}$ with self intersection -1 . Moreover if we assume that $M$ is a minimal surface and that $M$ is neither rational nor ruled, then $M$ is obtained from $\widetilde{M}$ by blowing down all holomorphic sections of $f: \widetilde{M} \rightarrow \boldsymbol{P}^{1}$ of self intersection -1 . We do not know whether every continuous section with self intersection -1 is homotopic to 
a holomorphic section. This leads to the following problem:

Problem 3.1. Is the differentiable structure of an algebraic surface $M$ (minimal, not rational or ruled) determined by an admissible sequence of vanishing cycles arising from a Lefschetz pencil?

Let $f_{1}: M_{1} \rightarrow S^{2}$ and $f_{2}: M_{2} \rightarrow S^{2}$ be two Lefschetz fibrations whose regular fibers are oriented surfaces of genus $g$. Choose regular fibers $f_{1}^{-1}(a) \in M_{1}$ and $f_{2}^{-1}(b) \in M_{2}$, and a diffeomorphism $\alpha: f_{1}^{-1}(a) \rightarrow$ $f_{2}^{-1}(b)$. We may construct a new Lefschetz fibration $f_{1} \sharp_{\alpha} f_{2}: M_{1} \#_{\alpha} M_{2} \rightarrow S^{2}$ as follows: Let $T_{1} \cong f_{1}^{-1}(a) \times D^{2}$ be a tubular neighborhood of $f_{1}^{-1}(a)$ in $M_{1}$, and let $T_{2} \cong f_{2}^{-1}(b) \times D^{2}$ be a tubular neighborhood of $f_{2}^{-1}(b)$ in $M_{2}$. Then $M_{1} \#_{\alpha} M_{2}$ is the union of $M_{1}$-int $\left(T_{1}\right)$ with $M_{2}$-int $\left(T_{9}\right)$ where we identify the boundaries by the mapping $f_{1}^{-1}(a) \times S^{1} \rightarrow$ $f_{2}^{-1}(b) \times S^{1}$ which sends $(x, z)$ to $(\alpha(x), \bar{z})$. The mapping $f_{1} \# f_{2}$ is defined in an obvious way. $f_{1} \# f_{2}: M_{1} \#_{\alpha} M_{9} \rightarrow S^{2}$ will be called a fiber connected sum of $f_{1}: M_{1} \rightarrow S^{2}$ and $f_{2}: M_{2} \rightarrow S^{2}$. In general $M_{1} \#_{\alpha} M_{2}$ will depend on the diffeomorphism $\alpha$. It is not hard to see that if $\alpha, \beta: f_{1}^{-1}(a) \rightarrow f_{2}^{-1}(b)$ are two diffeomorphisms, then $M_{1} \#_{\alpha} M_{2}$ is equivalent (in the sense of Lefschetz fibrations) to $M_{1} \#_{\beta} M_{2}$ if either $\alpha^{-1} \circ \beta: f_{1}^{-1}(a) \rightarrow f_{1}^{-1}(a)$ is in the image of the (geometric) monodromy group of $M_{1}$, or if $\alpha \circ \beta^{-1}: f_{2}^{-1}(b) \rightarrow f_{2}^{-1}(b)$ is in the image of the (geometric) monodromy group of $M_{2}$.

DEFinition 3.2. We will say that a Lefschetz fibration $f: M \rightarrow S^{2}$ is irreducible if it is not equivalent to a fiber connected sum $f_{1} \# f_{2}: M_{1} \#_{\alpha} M_{2} \rightarrow S^{2}$ where both $f_{1}$ and $f_{2}$ have at least one critical point. Algebraically, an irreducible Lefschetz fibration with regular fiber $X$ is determined by an equation: $\delta_{\mu} \cdots \delta_{1}=1$ in the mapping class group of $X$ such that $\delta_{\mu^{\prime}} \cdots \delta_{1}$ is not equivalent (in the sense given at the end of $\S 2)$ to $\delta_{\mu}^{\prime} \cdots \delta_{\nu+1}^{\prime} \delta_{\nu}^{\prime} \cdots \delta_{1}^{\prime}$ where $\delta_{\nu}^{\prime} \circ \cdots \circ \delta_{1}^{\prime}=1$ and $\delta_{\prime ! \prime}^{\prime} \circ \cdots \circ \delta_{\nu+1}^{\prime}=1$.

Problem 3.3. For a given surface $X$ and integer $\mu>0$, are there only finitely many (up to equivalence) irreducible Lefschetz fibrations $f: M \rightarrow S^{2}$ with $/$ vanishing cycles.

REMARK 3.4. The result of Moishezon (Example 2.5) states that if genus $X=1$, then there is only one irreducible Lefschetz fibration $f: M \rightarrow S^{2}$ where the number $\mu$ of critical points, is 12 . In this case, every element of the mapping class group of $X$ is realized by a geometric monodromy, and therefore every Lefschetz fibration of genus 1 is equivalent to $M \# \cdots \# M$.

If the answer to problem 3.1 is "yes", we would like to use Lefschetz pencils to study the diffeomorphism types of algebraic 
surfaces. Specifically, let $M$ be an algebraic surface, minimal, not rational or ruled, and let $f: \widetilde{M} \rightarrow \boldsymbol{P}^{1}$ be a Lefschetz fibration arising from a Lefschetz pencil on $M$. We have given a procedure for obtaining a handlebody decomposition of $\widetilde{M}$. We would like to have such a decomposition for $M$. This could be done if it were possible to rearrange our handlebody decomposition, by sliding handles, so that the sections of $f: \widetilde{M} \rightarrow \boldsymbol{P}^{1}$ of self intersection -1 would occur as the cores of 2 -handles.

Problem 3.4. Can the handlebody decomposition of $f: \check{M}, \boldsymbol{P}^{1}$ be altered so as to obtain a handlebody decomposition of $M$ ?

\section{REFERENCES}

1. A. Andreotti and T. Frankel, The Second Lefschetz Theorem on Hyperplane Section, Global Analysis, Princeton University Press (1969), 1-20.

2. A. Douady, Arrondissement des Arrêtes, Sem. H. Cartan, 14 (1961/62), No. 3.

3. Deligne and Katz, Groupes de Monodromie en Géométrie Algébrique, Lecture Notes in Math. 340, Springer-Verlag.

4. C. J. Earle and J. Eells, The diffeomorphism group of a compact Riemann surface, Bull. Amer. Math. Soc., 73 No. 4 (1967), 557-560.

5. Harer, Kas and Kirby to appear.

6. R. Mandelbaum and B. Moishezon, On the Topological Structure of Non-Singular Algebraic Surfaces in CP CPology, $^{3}$ T (1976), 23-40.

7. R. Mandelbaum, Special handlebody decompositions of simply connected algebraic surfaces, to appear.

8. J. Milnor, Morse theory, Annals of Math., 51, Princeton Univ. Press, (1963).

9. - Lectures on the h-cobordism theorem, Princeton Mathematical Notes, (1965).

10. B. Moishezon, Complex Surfaces and Connected Sums of Complex Projective Planes, Lecture Notes in Math., 603 Springer-Verlag.

11. S. Smale, Generalized Poincaré's conjecture in dimensions greater than Four, Ann. of Math., 74 No. 2 (1961).

Received November 1, 1978. During the preparation of this work, the author was partly supported by N.S.F., Grant MCS77-05583.

Oregon State University

CoRvallis, OR 97331 


\section{PACIFIC JOURNAL OF MATHEMATICS}

\section{EDITORS}

DONALD BABBITT (Managing Editor)

University of Galifornia

Los Angeles, California 90024

Hugo RossI

University of Utah

Salt Lake City, UT 84112

C. C. MoOre AND ANDrew OGG

University of California

Berkeley, CA 94720

\section{J. DugundjI}

Department of Mathematics University of Southern California

Los Angeles, California 90007

R. Finn and J. Milgram

Stanford University

Stanford, California 94305

ASSOCIATE EDITORS

E. F. Beckenbachi

B. H. NeumanN

F. WOLF

K. YOSHIDA

\section{SUPPORTING INSTITUTIONS}

UNIVERSITY OF BRITISH COLUMBIA

CALIFORNIA INSTITUTE OF TECHNOLOGY

UNIVERSITY OF CALIFORNIA

MONTANA STATE UNIVERSITY

UNIVERSITY OF NEVADA, RENO

NEW MEXICO STATE UNIVERSITY

OREGON STATE UNIVERSITY

UNIVERSITY OF OREGON
UNIVERSITY OF SOUTHERN CALIFONIA

STANFORD UNIVERSITY

UNIVERSITY OF HAWAII

UNIVERSITY OF TOKYO

UNIVERSITY OF UTAH

WASHINGTON STATE UNIVERSITY

UNIVERSITY OF WASHINGTON

Printed in Japan by International Academic Printing Co., Ltd., Tokyo, Japan 


\section{Pacific Journal of Mathematics \\ Vol. 89 , No. 1 \\ May, 1980}

David Bressoud, A note on gap-frequency partitions ................ 1

John David Brillhart, A double inversion formula ................. 7

Frank Richard Deutsch, Günther Nürnberger and Ivan Singer, Weak

Chebyshev subspaces and alternation .......................

Edward Richard Fadell, The relationship between Ljusternik-Schnirelman

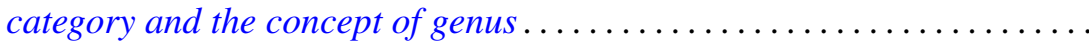

Harriet Jane Fell, On the zeros of convex combinations of polynomials.......

John Albert Fridy, An addendum to: "Tauberian theorems via block

dominated matrices" ..................................

Andrzej Granas, Ronald Bernard Guenther and John Walter Lee, Applications of topological transversality to differential equations. I. Some nonlinear

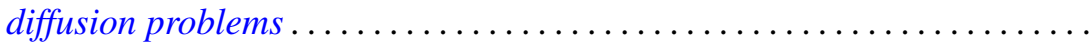

David E. Handelman and G. Renault, Actions of finite groups on self-injective

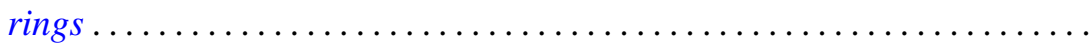

Michael Frank Hutchinson, Local $\Lambda$ sets for profinite groups .............

Arnold Samuel Kas, On the handlebody decomposition associated to a

Lefschetz fibration...

Hans Keller, On the lattice of all closed subspaces of a Hermitian space.....

P. S. Kenderov, Dense strong continuity of pointwise continuous

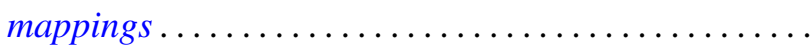

Robert Edward Kennedy, Krull rings.................

Jean Ann Larson, Richard Joseph Laver and George Frank McNulty,

Square-free and cube-free colorings of the ordinals ...

Viktor Losert and Harald Rindler, Cyclic vectors for $L^{p}(G)$

John Rowlay Martin and Edward D. Tymchatyn, Fixed point sets of

1-dimensional Peano continua...

Augusto Nobile, On equisingular families of isolated singularities ...

Kenneth Joseph Prevot, Imbedding smooth involutions in trivial bundles ...

Thomas Munro Price, Spanning surfaces for projective planes in four space.

Dave Riffelmacher, Sweedler's two-cocycles and Hochschild cohomology....

Niels Schwartz, Archimedean lattice-ordered fields that are algebraic over

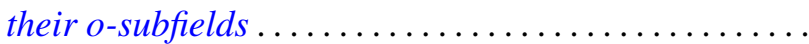

Chao-Liang Shen, A note on the automorphism groups of simple dimension groups.

Kenneth Barry Stolarsky, Mapping properties, growth, and uniqueness of

Vieta (infinite cosine) products ...

Warren James Wong, Maps on simple algebras preserving zero products. I.

The associative case ............................. 